\title{
A FORMULA FOR THE TANGENT BUNDLE OF FLAG MANIFOLDS AND RELATED MANIFOLDS
}

\author{
BY \\ KEE YUEN LAM( ${ }^{1}$ )
}

\begin{abstract}
A formula is given for the tangent bundle of a flag manifold $G$ in terms of canonically defined vector bundles over $G$. The formula leads to a unified proof of some parallelizability theorems of Stiefel manifolds. It can also be used to deduce some immersion theorems for flag manifolds.
\end{abstract}

1. Introduction. In this note $\mathbf{F}$ will denote either the field $\mathbf{R}$ of real numbers, the field $\mathbf{C}$ of complex numbers, or the skew-field $\mathbf{H}$ of quaternions. $Z(F)$ will denote the centre of $\mathbf{F}$, and $d=\operatorname{dim}_{\mathrm{R}} \mathbf{F}$. The space $\mathbf{F}^{n}$ of all $n$-tuples of elements in $\mathbf{F}$ will be equipped with a "hermitian" product $\langle$,$\rangle with values in \mathbf{F}$, and a dot product with values in $\mathrm{R}$, as follows: if $x=\left(x_{1}, \ldots, x_{n}\right), y=\left(y_{1}\right.$, $\ldots, y_{n}$ ) are in $\mathrm{F}^{n}$, then

$$
\begin{aligned}
& \langle x, y\rangle=x_{1} \bar{y}_{1}+x_{2} \bar{y}_{2}+\cdots+x_{n} \bar{y}_{n} \in \mathbf{F}, \\
& x \cdot y=1 / 2(\langle x, y\rangle+\langle y, x\rangle) \in \mathbf{R} .
\end{aligned}
$$

Finally, all vector spaces over $\mathbf{F}$ are left vector spaces, unless otherwise specified.

Suppose now $n_{1}, n_{2}, \ldots, n_{s}$ are fixed positive integers such that $n_{1}+$ $n_{2}+\cdots+n_{s}=n$. By a "flag", or more precisely a " $\left(n_{1}, \ldots, n_{s}\right)$-flag over F", we mean a collection $\sigma$ of mutually orthogonal subspaces $\left(\sigma_{1}, \ldots, \sigma_{s}\right)$ in $F^{n}$ such that $\operatorname{dim}_{F} \sigma_{i}=n_{i}$. The space of all such flags forms a compact smooth manifold (and a complex manifold in case $\mathbf{F}=\mathbf{C}$ ). We shall denote this manifold by $G_{\mathrm{F}}\left(n_{1}, \ldots, n_{s}\right)$, or simply by $G$ if $n_{1}, \ldots, n_{s}$ and $\mathbf{F}$ are clearly understood. As an example, note that $G_{\mathrm{R}}\left(n_{1}, n_{2}\right)$ is just the Grassmannian manifold of $n_{1}$ planes in Euclidean $n_{1}+n_{2}$ space.

One way to describe $G$ as a smooth manifold is the following. Let $U_{\mathrm{F}}(n)$ be the Lie group of all $n \times n$ matrices $A$ with entries in $\mathrm{F}$ satisfying $A \bar{A}^{t}=I_{n}$. Then $U_{\mathrm{F}}(n)$ acts on $\mathrm{F}^{n}$ from the right, the action being $(x, A) \mapsto x A$, where $x \in \mathrm{F}^{n}$ is regarded as a row vector. In a well-known manner, one can identify

Received by the editors August 14, 1974.

AMS (MOS) subject classifications (1970). Primary 55F25; Secondary 57D25, 57D40.

Key words and phrases. Real, complex and quaternionic flag manifolds, tangent bundle, canonical vector bundles over flag manifolds, parallelizability of Stiefel manifolds, projective Stiefel manifolds, immersion of flag manifolds into Euclidean spaces.

(1) Supported by the National Research Council of Canada, research grant A7562. 
the space $G$ with the homogeneous space $U_{F}(n) / U_{F}\left(n_{1}\right) \times \cdots \times U_{F}\left(n_{s}\right)$. In particular, $G$ is a compact smooth manifold, whose dimension can be computed to be $1 / 2 d\left(n^{2}-\Sigma n_{i}^{2}\right)$.

The purpose of this note is to furnish an explicit formula for the tangent bundle of $G$, and to derive from it some applications to questions such as immersing $G$ into Euclidean spaces, parallelizability of Stiefel manifolds, etc.

We begin by observing that there are naturally defined F-vector bundles $\xi_{1}, \xi_{2}, \ldots, \xi_{s}$ over $G$. In fact we just define $\xi_{i}$ to be the F-vector bundle whose fibre at the point $\sigma$ is the vector space $\sigma_{i}$. Note that since $\sigma_{i}$ inherits the hermitian product of $\mathbf{F}^{n}, \xi_{i}$ is automatically a $\mathbf{F}$-vector bundle with "hermitian" product $\langle$,$\rangle . Next it should be noted that there is an F-isomorphism$

$$
\xi_{1} \oplus \xi_{2} \oplus \cdots \oplus \xi_{s} \approx_{F} n \epsilon_{F} \quad \text { (= trivial bundle of F-dim } n \text { ), }
$$

because $\xi_{1} \oplus \cdots \oplus \xi_{s}$ is obtained by simply "erecting $\mathbf{F}^{n}$ as fibre over each and every point $\sigma \in G$." Furthermore, this isomorphism preserves hermitian product.

THEOREM (1.1). The tangent bundle $T(G)$ of $G$ is isomorphic to

$$
\bigoplus_{1 \leqslant i<j \leqslant s} \operatorname{Hom}_{F}\left(\xi_{i}, \xi_{j}\right)
$$

as $Z(\mathbf{F})$-vector bundles.

From elementary linear algebra one has

$$
\operatorname{Hom}_{F}\left(\xi_{i}, \xi_{j}\right) \approx_{Z(F)} \operatorname{Hom}_{F}\left(\xi_{i}, \epsilon_{F}\right) \otimes_{F} \xi_{j},
$$

where $\epsilon_{\mathrm{F}}$ is the trivial bundle $G \times \mathbf{F}$ and $\operatorname{Hom}_{\mathrm{F}}\left(\xi_{i}, \epsilon_{\mathrm{F}}\right)$ is the dual bundle of right F-vector spaces. Furthermore, the hermitian product in $\xi_{i}$ permits us to identity $\operatorname{Hom}_{F}\left(\xi_{i}, \epsilon_{F}\right)$ with the conjugate bundle $\bar{\xi}_{i}$, whose typical fibre is the right $F$ vector space obtained by forming the "conjugate" of the corresponding fibre of $\xi_{i}$ (see §4). Thus theorem (1.1) can be stated as

COROLlaRY (1.2). The tangent bundle $T(G)$ of $G$ is isomorphic to

$$
\bigoplus_{1<i<j \leqslant s} \bar{\xi}_{i} \otimes_{F} \xi_{j}
$$

as $Z(\mathbf{F})$-vector bundles.

2. Proof of Theorem (1.1). To prove Theorem (1.1) we first show that for each $i<j, \operatorname{Hom}_{\mathrm{F}}\left(\xi_{i}, \xi_{j}\right)$ can be embedded into $T(G)$ as a $Z(\mathbf{F})$-subbundle in a natural way. For notational convenience we shall describe the embedding of $\operatorname{Hom}_{F}\left(\xi_{1}, \xi_{2}\right)$ into $T(G)$, the description for the other embeddings being entirely similar.

Let $\sigma=\left(\sigma_{1}, \sigma_{2}, \ldots, \sigma_{s}\right)$ be a flag, or a point in $G$. The fibre of 
$\operatorname{Hom}_{\mathrm{F}}\left(\xi_{1}, \xi_{2}\right)$ over $\sigma$ is the vector space $\operatorname{Hom}_{\mathrm{F}}\left(\xi_{1}, \xi_{2}\right)_{\sigma}=\operatorname{Hom}_{\mathrm{F}}\left(\sigma_{1}, \sigma_{2}\right)$. Let $f: \sigma_{1} \rightarrow \sigma_{2}$ be an element of this fibre. For any $t \in \mathbf{R}$ let $\gamma(t f)$ be the graph of tf in the direct sum $\sigma_{1} \oplus \sigma_{2}$, and let $\gamma^{\perp}(t f)$ be the orthogonal complement of $\gamma(t f)$ in this direct sum. Clearly, $\gamma(t f)$ is an $n_{1}$-plane in $F^{n}$, and coincides with $\sigma_{1}$ when $t=0$. As $t$ varies, the flag

$$
\sigma_{t}=\left(\gamma(t f), \gamma^{\perp}(t f), \sigma_{3}, \ldots, \sigma_{s}\right)
$$

describes a smooth curve in $G$ passing through $\sigma$, and we can take its tangent vector $(f)$ at $\sigma$.

LEMMA (2.1). The correspondence $f \rightarrow \iota(f)$ induces an embedding $\iota$ of $\operatorname{Hom}_{\mathrm{F}}\left(\xi_{1}, \xi_{2}\right)$ into $T(G)$.

To prove Lemma (2.1) we shall introduce coordinates in a neighbourhood of the point $\sigma$, as follows. By recoordinating $\mathrm{F}^{n}$ if necessary, one can suppose that the first $n_{1}$ rows of the identity matrix $I_{n}$ form a basis of $\sigma_{1}$, the next $n_{2}$ rows of $I_{n}$ form a basis of $\sigma_{2}$, and so on. Consider now a matrix

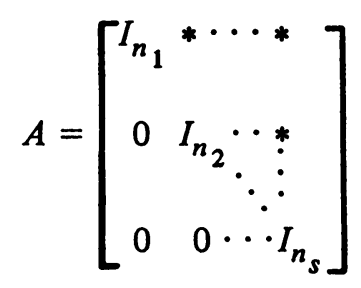

in upper diagonal block form with entries in $\mathbf{F}$. Let $V_{i} \subset F^{n}$ be the subspace spanned by the first $n_{1}+n_{2}+\cdots+n_{i}$ rows of $A$. Let $\sigma_{i}^{A}$ be the orthogonal complement of $V_{i-1}$ in $V_{i}$. Then $\sigma(A)=\left(\sigma_{1}^{A}, \sigma_{2}^{A}, \ldots, \sigma_{s}^{A}\right)$ is a flag, and hence a point in $G$. Furthermore if $B$ is another upper diagonal block matrix, then $\sigma(A)=\sigma(B)$ implies $A=B$. Thus we can use the upper diagonal entries of $A$ as a coordinate chart (a complex coordinate chart in case $\mathbf{F}=\mathbf{C}$ ) for an open neighbourhood $U$ of the point $\sigma \in G$, with $\sigma$ being the origin.

Using the bases of $\sigma_{1}$ and $\sigma_{2}$ given above, we can write down the $n_{1} \times n_{2}$ matrix $M(f)$ of $f: \sigma_{1} \rightarrow \sigma_{2}$. If now

$$
A_{t}=\left[\begin{array}{cccc}
I_{n_{1}} & t M(f) & \cdots & 0 \\
0 & I_{n_{2}} & \cdots & 0 \\
\cdots & \cdots & \cdots & \cdots \\
0 & 0 & \cdots & I_{n_{s}}
\end{array}\right],
$$

then the curve $\sigma_{t}$ is just $\sigma\left(A_{t}\right)$,so that in terms of coordinates the components of the tangent vector $\iota(f)=\left(d \sigma_{t} / d t\right)_{t=0}$, when arranged in blocks in upper diagonal positions, are simply 


$$
\begin{aligned}
& 0 \quad M(f) \cdots 0 \\
& 0 \cdots 0 \\
& \cdots 0
\end{aligned}
$$

This clearly shows that $\iota$ is a $Z(F)$-linear embedding of $\operatorname{Hom}_{F}\left(\xi_{1}, \xi_{2}\right)$ into $T(G)$.

To finish the proof of Theorem (1.1), let $\iota_{i, j}(i<j)$ be the natural embedding of $\operatorname{Hom}_{\mathrm{F}}\left(\xi_{i}, \xi_{j}\right)$ into $T(G)$. If $f_{i, j}: \sigma_{i} \rightarrow \sigma_{j}$ is an arbitrary vector in the fibre of $\operatorname{Hom}_{F}\left(\xi_{i}, \xi_{j}\right)$ over $\sigma$, then in the coordinate neighbourhood $U$, the components for the tangent vector $\Sigma_{1 \leqslant i<j \leqslant s} l_{i, j}\left(f_{i, j}\right)$, when arranged in blocks in upper diagonal positions, are just

$$
\begin{array}{rll}
M\left(f_{1,2}\right) M\left(f_{1,3}\right) & \cdots & M\left(f_{1, s}\right) \\
M\left(f_{2,3}\right) & \cdots & M\left(f_{2, s}\right) \\
& \cdots & M\left(f_{s-1, s}\right) .
\end{array}
$$

This makes it clear that

$$
\bigoplus_{1 \leqslant i<j \leqslant s} \operatorname{Hom}_{\mathrm{F}}\left(\xi_{i}, \xi_{j}\right) \approx_{Z(\mathrm{~F})} T(G),
$$

and Theorem (1.1) is proved.

REMARK. In the case of Grassmannian manifolds, the formula for $T(G)$ has been established in [9, p. 411] for $\mathbf{F}=\mathbf{R}$, and also in [5, p. 217] using Lie group representations. (See also [8].) Our formula for the tangent bundle of flag manifolds can be regarded both as an extension to that of [9], and as a totally different approach in the Grassmannian case that is considerably simpler than the treatment of [5].

3. Tangent bundle of some related manifolds. It is easy to see that there is an analogous formula for the tangent bundle of manifolds formed by the set of all mutually orthogonal subspaces $\left(\hat{\sigma}_{1}, \hat{\sigma}_{2}, \ldots, \hat{\sigma}_{k}, \sigma_{k+1}, \ldots, \sigma_{s}\right)$ in $\mathbf{R}^{n}$ with R-dimensions equal to $n_{1}, n_{2}, \ldots, n_{s}$ respectively, and with $\hat{\sigma}_{1}, \ldots, \hat{\sigma}_{k}$ oriented. In particular, real Stiefel manifolds can be looked upon as manifolds of this type. However, we shall give a unified treatment for the tangent bundle of real, complex and quaternionic Stiefel manifolds in the next paragraph.

By the Stiefel manifold $V=V_{\mathrm{F}}(n, k)$ we mean the manifold of all $k$-frames $\theta$ in $\mathrm{F}^{n}$ whose $k$ vectors $\theta_{1}, \theta_{2}, \ldots, \theta_{k}$ satisfy $\left\langle\theta_{i}, \theta_{j}\right\rangle=\delta_{i j}$ for $1 \leqslant i, j \leqslant k$. Alternatively we can define $V$ to be the homogeneous space $U_{\mathrm{F}}(n) / U_{\mathrm{F}}(n-k)$. If $\theta \in V$ is a $k$-frame in $\mathbf{F}^{n}$, let $\mathbf{F} \theta_{i}$ be the $\mathbf{F}$-subspace spanned by the $i$ th vector $\theta_{i}$ of the frame $\theta$, and let $\theta^{\perp}$ be the orthogonal complement of $\mathbf{F} \theta_{1} \oplus \cdots \oplus \mathbf{F} \theta_{k}$ in $\mathbf{F}^{n}$. Then $\pi(\theta)=\left(\mathbf{F} \theta_{1}, \ldots, \mathbf{F} \theta_{n}, \theta^{\perp}\right)$ is a $(1, \ldots, 1, n-k)$-flag in $\mathbf{F}^{n}$, and in this way we obtain a principal fibration 


$$
\pi: V_{\mathrm{F}}(n, k) \rightarrow G_{\mathrm{F}}(\underbrace{1, \ldots, n-k),}_{k}
$$

whose fibre is $U_{\mathrm{F}}(1) \times \cdots \times U_{\mathrm{F}}(1)$ ( $k$ factors). Furthermore, if $\xi_{1}, \ldots, \xi_{k}$ and $\xi_{k+1}$ are the canonical bundles over $G_{\mathrm{F}}(1, \ldots, 1, n-k)$ defined in $\S 1$, then the pull-back bundles $\pi^{*}\left(\xi_{i}\right)$ have the following description:

(1) For $1 \leqslant i \leqslant k, \pi^{*}\left(\xi_{i}\right)$ is the trivial bundle $\epsilon_{\mathrm{F}}$. This is because the fibre $\pi^{*}\left(\xi_{i}\right)_{\theta}$ is none other than $\mathbf{F} \theta_{i}$, in which the vector $\theta_{i}$ serves as a canonical nonzero section.

(2) $\pi^{*}\left(\xi_{k+1}\right)$ is obtained by "erecting $\theta^{\perp}$ as the fibre over $\theta \in V_{\mathrm{F}}(n, k)$ ". Henceforth, we shall refer to this bundle as $\eta_{\mathrm{F}}(n, k)$, or simply as $\eta$ if $n, k$ and $\mathbf{F}$ are clearly understood.

(3) Since $\xi_{1} \oplus \cdots \oplus \xi_{k} \oplus \xi_{k+1} \approx_{F} n \epsilon_{F}$, applying $\pi^{*}$ gives $k \epsilon_{F} \oplus \eta \approx_{F}$ $n \epsilon_{\mathrm{F}}$, or $k d \epsilon_{\mathrm{R}} \oplus \eta \approx n d \epsilon_{\mathrm{R}}$ if the underlying real bundle of $\eta$ is to be emphasised.

In particular, this shows that

$$
l \epsilon_{\mathrm{R}} \oplus \eta \approx \text { trivial bundle, whenever } l \geqslant k d .
$$

The above information now enables us to prove

THEOREM (3.1). For $n \geqslant k \geqslant 2$, the Stiefel manifold $V_{\mathbf{F}}(n, k)$ is parallelizable, i.e. its tangent bundle is R-trivial.

Proof. Since $\pi$ is a principal fibration, the bundle along the fibre is the trivial bundle $k(d-1) \epsilon_{\mathbf{R}}$. Hence, with " $\approx$ " meaning "isomorphisms over $\mathbf{R}$ ",

$$
\begin{aligned}
T\left(V_{\mathrm{F}}(n, k)\right) & \approx \pi^{*}\left(T\left(G_{\mathrm{F}}(1, \ldots, 1, n-k)\right)\right) \oplus \text { bundle along the fibre } \\
& \approx \bigoplus_{1 \leqslant i<j \leqslant k+1} \pi^{*}\left(\operatorname{Hom}_{\mathrm{F}}\left(\xi_{i}, \xi_{j}\right)\right) \oplus k(d-1) \epsilon_{\mathrm{R}} \\
& \approx \bigoplus_{1 \leqslant i<j \leqslant k} \operatorname{Hom}_{\mathrm{F}}\left(\epsilon_{\mathrm{F}}, \epsilon_{\mathrm{F}}\right) \oplus k \operatorname{Hom}_{\mathrm{F}}\left(\epsilon_{\mathrm{F}}, \eta\right) \oplus k(d-1) \epsilon_{\mathrm{R}} \\
& \approx 1 / 2 k(k-1) d \epsilon_{\mathrm{R}} \oplus k \eta \oplus k(d-1) \epsilon_{\mathrm{R}} \\
& \approx 1 / 2 k(k d+d-2) \epsilon_{\mathrm{R}} \oplus k \eta .
\end{aligned}
$$

Since $1 / 2 k(k d+d-2) \geqslant k d$ unless $\mathbf{F}=\mathbf{R}$ and $k=2$, we can repeatedly apply (*) to conclude that, apart from $V_{\mathrm{R}}(n, 2), T\left(V_{\mathrm{F}}(n, k)\right)$ is trivial for all $k \geqslant 2$. As for $V_{\mathbf{R}}(n, 2)$, our argument only shows that $T\left(V_{\mathbf{R}}(n, 2)\right) \oplus \epsilon_{\mathbf{R}}$ is trivial. Although one can refine the argument to trivialise $T\left(V_{R}(n, 2)\right)$ in case $n \equiv 0(\bmod 4)$, using the fact that $S^{n-1}$ has three vector fields that lift to three sections in $\eta \oplus \epsilon_{\mathrm{R}}$, the parallelizability of $V_{\mathbf{R}}(n, 2)$ for general $n$ seems to require a different type of argument, such as for example in [2] or [10].

REMARK. The parallelizability of $V_{\mathbf{F}}(n, k)$ for $k \geqslant 2$ is a known result, 
see Sutherland [10] and Handel [6]. Nevertheless, the proof given in Theorem (3.1) is more elementary and unifies the cases $k \geqslant 3$ for all possible $\mathbf{F}$. It also provides a rather explicit trivialisation of the tangent bundle of Stiefel manifolds, save for the exceptional case of $V_{R}(n, 2)$.

We now confine ourselves to $\mathbf{F}=\mathbf{R}$ or $\mathbf{C}$, and turn our attention to the so called "projective Stiefel manifolds", which have found some applications in topology (see [1], [3] and [4]). The projective Stiefel manifold $P V_{F}(n, k)$, by definition, is obtained from $V_{F}(n, k)$ by identifying any frame $\theta=\left(\theta_{1}, \ldots, \theta_{k}\right)$ with the frame $\alpha \theta=\left(\alpha \theta_{1}, \ldots, \alpha \theta_{k}\right)$ for any $\alpha \in U_{F}(1)$. For convenience, we can refer to a point $[\theta]$ in $P V_{F}(n, k)$ as a "projective $k$-frame" in $F^{n}$. Note that as a homogeneous space, $P V_{F}(n, k)$ is just $U_{F}(n) / \Delta_{F}(k) \times U_{F}(n-k)$, where $\Delta_{F}(k)$ is the subgroup of $U_{F}(1) \times \cdots \times U_{F}(1)$ consisting of matrices $\alpha I_{k}$ with $\alpha \in U_{F}(1)$. There is a projection map

$$
p: P V_{\mathbf{F}}(n, k) \rightarrow G_{\mathbf{F}}(1, \ldots, 1, n-k)
$$

obtained by sending a projective frame $[\theta]$ to the flag $\left(\mathbf{F} \theta_{1}, \ldots, \mathbf{F} \theta_{k}, \theta^{\perp}\right)$. Note that $p$ is a principal fibration with structural group $U_{\mathrm{F}}(1) \times \cdots \times U_{\mathrm{F}}(1) / \Delta_{\mathrm{F}}(k)$. (Remember that $U_{\mathbf{F}}(1)$ is communative for $\mathbf{F}=\mathbf{R}$ or $\mathbf{C}$.) Under $p$, the pull-back bundles $p^{*}\left(\xi_{i}\right), 1 \leqslant i \leqslant k+1$, have the following description:

(1) If $\zeta$ is the $\mathbf{F}$-line bundle over $P V_{\mathrm{F}}(n, k)$ well defined by erecting $\mathbf{F} \theta_{1}$ as fibre over the projective frame $[\theta]=\left[\theta_{1}, \ldots, \theta_{k}\right]$, then for $1 \leqslant i \leqslant k$, $p^{*}\left(\xi_{i}\right) \approx_{\mathrm{F}} \zeta$ as $\mathbf{F}$-vector bundles. This is because the map

$$
p^{*}\left(\xi_{i}\right)_{[\theta]} \ni \lambda \theta_{i} \mapsto \lambda \theta_{1} \in \zeta_{[\theta]} \quad(\lambda \in \mathrm{F})
$$

gives a well-defined $\mathbf{F}$-isomorphism from $p^{*}\left(\xi_{i}\right)$ to $\zeta$.

(2) $p^{*}\left(\xi_{k+1}\right)$ is the bundle which erects the vector space $\theta^{\perp}$ as fiber over the projective frame $[\theta]$. We shall also denote this bundle by $\eta$, for the reason that under the identification map $V_{\mathrm{F}}(n, k) \rightarrow P V_{\mathrm{F}}(n, k)$, it pulls back to what we previously called $\eta$ over the Stiefel manifold $V_{\mathrm{F}}(n, k)$.

Since $p$ is a principal fibration, the bundle along the fibre for $p$ is trivial, of R-dimension $(k-1)(d-1)$. Consequently, the tangent bundle of a projective Stiefel manifold as a real bundle is given by

$$
\begin{aligned}
T\left(P V_{\mathrm{F}}(n, k)\right) & \approx p^{*} T(G(1, \ldots, 1, n-k)) \oplus(k-1)(d-1) \epsilon_{\mathrm{R}} \\
& \approx p^{*}\left(\bigoplus_{1 \leqslant i<j \leqslant k+1}^{\oplus} \operatorname{Hom}_{\mathrm{F}}\left(\xi_{i}, \xi_{j}\right)\right) \oplus(k-1)(d-1) \epsilon_{\mathrm{R}} \\
& \approx \bigoplus_{1<i<j<k} \operatorname{Hom}_{\mathrm{F}}(\zeta, \zeta) \oplus k \operatorname{Hom}_{\mathrm{F}}(\zeta, \eta) \oplus(k-1)(d-1) \epsilon_{\mathbf{R}} .
\end{aligned}
$$

Since $\operatorname{Hom}_{\mathbf{F}}(\zeta, \zeta)$ is trivial for $\mathbf{F}=\mathbf{R}$ or $\mathbf{C}$, we obtain 
THEOREM (3.2). The tangent bundle of a projective Stiefel manifold is given by

$$
T\left(P V_{\mathbf{F}}(n, k)\right) \approx k \operatorname{Hom}_{\mathbf{F}}(\zeta, \eta) \oplus 1 / 2(k-1)(k d+2 d-2) \epsilon_{\mathbf{R}} .
$$

COROLlary (3.3). This tangent bundle, abbreviated as $T$, satisfies the relationship $T \oplus(1 / 2 k(k-1) d+k+d-1) \epsilon_{\mathrm{R}} \approx k n \zeta$.

PROoF. The relationship $\xi_{1} \oplus \cdots \oplus \xi_{k} \oplus \xi_{k+1} \approx_{F} n \epsilon_{F}$ pulls back under $p^{*}$ to give $k \zeta \oplus \eta \approx_{\mathrm{F}} n \epsilon_{\mathrm{F}}$. Multiplying by $k$, one obtains $k^{2} \zeta \oplus k \eta \approx_{\mathrm{F}} k n \epsilon_{\mathrm{F}}$. Taking $\operatorname{Hom}_{F}\left(\zeta,{ }_{-}\right)$, using $\operatorname{Hom}_{F}(\zeta, \zeta) \approx d \epsilon_{R}$ and $\operatorname{Hom}_{F}\left(\zeta, \epsilon_{F}\right) \approx_{R} \zeta$, we get

$$
k^{2} d \epsilon_{\mathrm{R}} \oplus k \operatorname{Hom}_{\mathrm{F}}(\zeta, \eta) \approx k n \zeta,
$$

from which the corollary follows by adding to both sides of $(* *)$ the trivial bundle $(1 / 2 k(k-1) d+k+d-1) \epsilon_{\mathrm{R}}$.

Note that when $k=1, P V_{\mathrm{F}}(n, 1)$ is just a real or complex projective space, and $\zeta$ is the canonical Hopf line bundle over it. In this case Corollary (3.3) simply reduces to the well-known facts about the tangent bundle of such spaces.

We remark finally that although an analogous manifold $P V_{\mathrm{F}}(n, k)$ can be defined for $\mathbf{F}$ the quaternions, the description of its tangent bundle is not as simple as in Theorem (3.2), due to the noncommutativity of the quaternions.

4. The functor $\mu^{2}$. In this section we discuss a functor $\mu^{2}$ analogous to the "symmetric power functor" $s^{2}$ on vector spaces. The result will be used in $\S 5$ to obtain immersions of flag manifolds into Euclidean spaces.

If $V$ is a left vector space over $\mathbf{F}$, the conjugate vector space $\bar{V}$ is the right F-vector space having $V$ as underlying abelian group, with scalar multiplication given by $v \alpha=\bar{\alpha} v$ for any $v \in V$ and $\alpha$ in $F$. The tensor product $\bar{V} \otimes_{F} V$ is then a vector space over $\mathbf{R}$, and the map $T: \bar{V} \otimes_{\mathrm{F}} V \rightarrow \bar{V} \otimes_{\mathrm{F}} V$ given by $T(u \otimes v)=$ $-v \otimes u$ is a well-defined, R-linear involution. (Although it is true that in case $\mathbf{F}=\mathbf{C}, \bar{V} \otimes_{\mathrm{F}} V$ is a $\mathbf{C}$-vector space and $T$ is actually conjugate linear, this additional structure will not be used in the sequel.) Let $\operatorname{Fix}(T)$ be the subspace of $\bar{V} \otimes_{F} V$ consisting of all fixed points of $T$. It can be verified that $\operatorname{Fix}(T)$ is spanned by tensors of the form $u \otimes v-v \otimes u$, together with those of the form $u \otimes \beta u$, where $\beta$ is any element in $F$ such that $\bar{\beta}=-\beta$. Define

$$
\mu_{\mathrm{F}}^{2}(V)=\bar{V} \otimes_{\mathrm{F}} V / \operatorname{Fix}(T) \text {. }
$$

Then $\mu_{\mathrm{F}}^{2}$ is a covariant functor from the category of left $\mathbf{F}$-vector spaces to the category of $\mathbf{R}$-vector spaces. Analogous to the property

$$
s^{2}\left(W \oplus W^{\prime}\right) \approx s^{2}(W) \oplus W \otimes W^{\prime} \oplus s^{2}\left(W^{\prime}\right)
$$

of the symmetric power functor $s^{2}$ for vector spaces over any field, we have 
Proposition (4.1). If $V, W$ are $\mathbf{F}$-vector spaces, then there is a natural isomorphism

$$
\mu_{\mathrm{F}}^{2}(V \oplus W) \approx \mu_{\mathrm{F}}^{2}(V) \oplus \bar{V} \otimes_{\mathrm{F}} W \oplus \mu_{\mathrm{F}}^{2}(W) .
$$

Proof. Consider the diagram

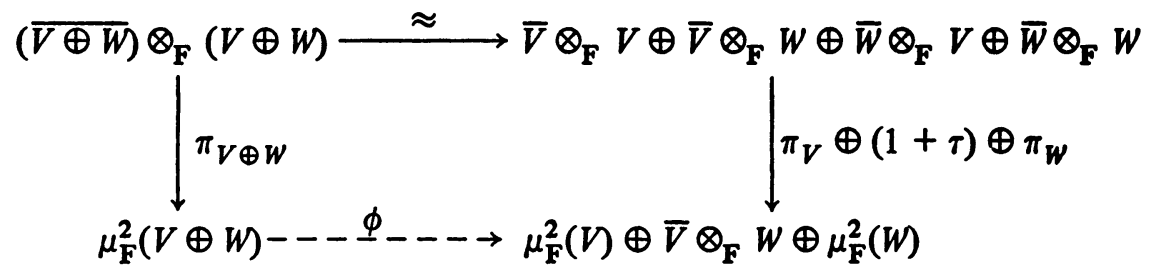

where the top map is the natural identification, the right vertical map is the direct sum of

$$
\pi_{V} \oplus \pi_{W}: \bar{V} \otimes_{\mathrm{F}} V \oplus \bar{W} \otimes_{\mathrm{F}} W \rightarrow \mu_{\mathrm{F}}^{2}(V) \oplus \mu_{\mathrm{F}}^{2}(W)
$$

with the map

$$
1+\tau: \bar{V} \otimes_{\mathbf{F}} W \oplus \bar{W} \otimes_{\mathbf{F}} V \rightarrow \bar{V} \otimes_{\mathbf{F}} W
$$

defined by $(1+\tau)\left(v \otimes w+w_{1} \otimes v_{1}\right)=v \otimes w+v_{1} \otimes w_{1}$. This right vertical map annihilates precisely $\operatorname{Fix}\left(T_{V \oplus W}\right)$, and so induces an isomorphism $\phi$ as indicated by the dotted arrow of the diagram.

CoRollary (4.2). If $\operatorname{dim}_{\mathrm{F}} V=m$, then $\operatorname{dim}_{\mathrm{R}} \mu^{2}(V)=1 / 2 m(m-1) d+m$.

ProoF. Under the identification $\overline{\mathbf{F}} \otimes_{\mathrm{F}} \mathbf{F} \approx \overline{\mathrm{F}}, T$ becomes the map $T(\alpha)=$ $-\bar{\alpha}$ for any $\alpha \in \overline{\mathbf{F}}$. Thus $\operatorname{Fix}(T)=\{\alpha \in \overline{\mathbf{F}} \mid \alpha=-\bar{\alpha}\}$, and so $\mu_{\mathbf{F}}^{2}(\mathbf{F})=\mathbf{R}$. The corollary then follows from Proposition (4.1) by induction on $m$.

Suppose now $V$ is equipped with a nondegenerate hermitian product $\langle$, over F. Since $\langle u, v\rangle=\langle\overline{v, u}\rangle$ and

$$
\langle u, \alpha v\rangle-\langle u \alpha, v\rangle=\langle u, v\rangle \bar{\alpha}-\bar{\alpha}\langle u, v\rangle
$$

for any $u, v \in V, \alpha \in \mathbf{F}$, the R-linear map $\bar{V} \otimes_{\mathbf{F}} V \rightarrow \mathbf{R}$ given by $u \otimes v \mapsto$ $u \cdot v=1 / 2(\langle u, v\rangle+\langle v, u\rangle)$ is well defined, and induces an epimorphism $j: \mu_{F}^{2}(V)$ $\rightarrow \mathbf{R}$. Such an epimorphism $j$ will be useful in $\$ 5$.

5. Immersion of flag manifolds into Euclidean spaces. The basic immersion theorem of $M$. Hirsch [7] states that if $M$ is an $m$-dimensional differentiable manifold with tangent bundle $T(M)$, such that there exists a real vector bundle $\nu$ of dimension $q>0$ over $M$ satisfying $\nu \oplus T(M)=(m+q) \epsilon_{\mathrm{R}}$, then $M$ immerses in $\mathbf{R}^{m+q}$. We shall now combine it with Theorem (1.1) to get immersion results for flag manifolds. 
THEOREM (5.1). The flag manifold $G=G_{\mathrm{F}}\left(n_{1}, \ldots, n_{s}\right)$ can be immersed in Euclidean space with codimension

$$
(n-s)+1 / 2 \sum_{i=1}^{s} n_{i}\left(n_{i}-1\right) d,
$$

provided this codimension is nonzero.

Proof. Take the isomorphism $\xi_{1} \oplus \cdots \oplus \xi_{s} \approx_{F} n \epsilon_{F}$, and apply the functor $\mu_{F}^{2}$ to both sides, using Proposition (4.1) along the way. The result is

$$
\bigoplus_{i=1}^{s} \mu_{\mathrm{F}}^{2}\left(\xi_{i}\right) \oplus\left(\underset{1 \leqslant i<j \leqslant s}{\bigoplus_{i}} \bar{\xi}_{i} \otimes_{\mathrm{F}} \xi_{j}\right) \approx \text { a trivial bundle. }
$$

By Corollary (1.2), the second summand on the left side is precisely the tangent bundle $T(G)$. Furthermore, since each $\xi_{i}$ is a bundle with nondegenerate hermitian product over $\mathbf{F}$, the final remark in $\S 4$ shows that there is a bundle epimorphism

$$
j: \bigoplus_{i=1}^{s} \mu_{\mathrm{F}}^{2}\left(\xi_{i}\right) \rightarrow s \epsilon_{\mathrm{R}}
$$

From these facts, it follows by standard argument that

$$
\operatorname{ker}(j) \oplus T(G) \approx \text { a trivial bundle. }
$$

Since the fibre dimension of $\operatorname{ker}(j)$ is $(n-s) \oplus \frac{1}{2} \Sigma_{i=1}^{s} n_{i}\left(n_{i}-1\right) d$, Hirsch's theorem can be applied to yield the desired immersions of $G$, proving Theorem (5.1).

In the case $\mathbf{F}=\mathbf{R}$, there is no need to distinguish a real vector bundle from its conjugate, so that $T(G)$ is simply $\bigoplus_{1 \leqslant i<j \leqslant s} \xi_{i} \otimes_{\mathrm{R}} \xi_{j}$. Using the second exterior power functor $\lambda^{2}$ instead of $\mu^{2}$, and arguing exactly the same way as in Theorem (5.1), one can get

COROllaRy (5.2). The real flag manifold $G_{\mathbf{R}}\left(n_{1}, \ldots, n_{s}\right)$ can be immersed in Euclidean space with codimension $1 / 2 \Sigma n_{i}\left(n_{i}-1\right)$, provided this codimension is nonzero.

This, of course, is a better result than the real case of Theorem (5.1).

In both the theorem and the corollary, if the codimension number turns out to be zero, then (\#) shows that the manifold in question (which is $G_{F}(1, \ldots, 1)$ ) must be stably parallelizable, and thus immerses in codimension one by Hirsch's theorem.

The above immersions are not always interesting. For example, if $G=$ $G_{\mathrm{F}}(1, n-1)$ is the projective space, then the immersion codimension far exceeds $\operatorname{dim} G$. However, there are cases in which the codimension is considerably less than $\operatorname{dim} G$ (such as when all $n_{i}$ 's are close to $n / s$ ). These then constitute the interesting cases of our theorem. 
REMARK. The immersion of complex flag manifolds is obtained by Tornehave [11], using a quite different method. To the best of our knowledge, the immersion results for quaternionic flag manifolds have not been given before in the literature.

\section{REFERENCES}

1. P. F. Baum and W. Browder, The cohomology of quotients of classical groups, Topology 3 (1965), 305-336. MR 32 \#6490.

2. G. E. Bredon and A. Kosinski, Vector fields on $\pi$-manifolds, Ann. of Math. (2) 84 (1966), 85-90. MR 34 \#823.

3. S. Gitler and D. Handel, The projective Stiefel manifolds. I, Topology 7 (1968), 39-46. MR 36 \#3373a.

4. S. Gitler, The projective Stiefel manifolds. II. Applications, Topology 7 (1968), 47-53. MR 36 \#3373b.

5. W. Greub, S. Halperin and R. Vanstone, Connection, curvature, and cohomology. Vol. II, Academic Press, New York, 1973.

6. D. Handel, $A$ note on the parallelizability of real Stiefel manifolds, Proc. Amer. Math. Soc. 16 (1965), 1012-1014. MR 32 \#457.

7. M. W. Hirsch, Immersions of manifolds, Trans. Amer. Math. Soc. 93 (1959), 242276. MR 22 \#9980.

8. W. C. Hsiang and R. H. Szczarba, On the tangent bundle of a Grassman manifold, Amer. J. Math. 86 (1964), 698-704. MR 30 \#2523.

9. I. R. Porteous, Topological geometry, Van Nostrand Reinhold, New York, 1969. MR 40 \#8059.

10. W. A. Sutherland, $A$ note on the parallelizability of sphere-bundles over spheres, J. London Math. Soc. 39 (1964), 55-62. MR 30 \#5327.

11. J. Tornehave, Immersions of complex flagmanifolds, Math. Scand. 23 (1968), 22-26 (1969). MR 40 \#4970.

DEPARTMENT OF MATHEMATICS, UNIVERSITY OF BRITISH COLUMBIA, VANCOUVER, BRITISH COLUMBIA, CANADA 